\title{
Attenuation limits in longitudinal phononic crystals
}

\author{
L Luschi $^{1}$, G Iannaccone ${ }^{1}$, F Pieri $^{1}$ \\ ${ }^{1}$ Dipartimento di Ingegneria dell'Informazione, Università di Pisa, Pisa, 56122 Italy
}

\begin{abstract}
The acoustic attenuation inside the bandgaps is, together with the bandgap width, a fundamental design parameter for phononic crystal-based systems. We discuss approximate expressions for the maximum attenuation inside the bandgaps of one-dimensional longitudinal phononic crystals and its dependence on the acoustic contrast and fractional bandwidth. We provide different approximations at small and large fractional bandwidths, computed from the trace of the transmission matrix of the crystal elementary cell. We show that, for relatively small gaps, the attenuation is roughly proportional to the fractional bandwidth, in analogy with the flexural case. For larger gaps, a large attenuation can only be obtained for high (and possibly impractical) acoustic contrasts. We also derive asymptotic upper limits for the bandgap borders and show that high contrasts do not necessarily lead to wide bandgaps, a fact connected to geometrical phase inversion for the acoustic wave in the crystal. We finally compare the attenuation of flexural and longitudinal waves at fixed fractional bandwidth and derive regions of optimum attenuation for the two propagation modes.
\end{abstract}

\section{Introduction}

Phononic crystals (PnCs) are structures with a periodic modulation of their mechanical properties: stiffness, mass, or both. The propagation of acoustic waves in a $\mathrm{PnC}$ is affected by this periodicity and shows a frequency-selective behavior. As a result, the frequency spectrum is divided into passbands, where the propagation is allowed, and bandgaps, where it is forbidden ${ }^{1}$.

PnCs with the simplest structure can be obtained by modulating the mechanical properties of a beam or bar structure, thereby obtaining a one-dimensional phononic crystal (1D PnC). One-dimensional crystals have been proposed in the context of several micro- and nano-electromechanical systems (M/NEMS) applications. MEMS-based PnC sensors exploiting both flexural ${ }^{2}$ and longitudinal ${ }^{3}$ modes have been proposed theoretically, PnC strips exhibiting full bandgaps have been used to suppress anchor losses in microelectromechanical (MEMS) resonators ${ }^{4}$, heterostructure-based flexural waveguides for NEMS circuits have been proposed ${ }^{5}$. At the nanometric scale, phonon-photon interaction has been demonstrated in the so-called optomechanical crystals ${ }^{6}$. Nanometric periodicity also meets the wavelength of thermal phonons, making 2D crystals with complete bandgaps suited for thermal conductivity suppression ${ }^{7,8}$. Furthermore, 2D crystals with complete bandgaps have also been used to realize cavity-based MEMS resonators ${ }^{9}$.

The introduction of a regular pattern of holes on the structural layer is the typical method to obtain a M/NEMS $\mathrm{PnC}^{2-10}$. In this context, both capacitive and piezoelectric actuation have been exploited. Air gaps at the end of a bar have been used for capacitive excitation of longitudinal waves ${ }^{3,10,11}$, while the same gaps placed under 
beam-like structures have served for excitation of flexural waves ${ }^{5}$. Piezoelectric transduction is another viable option, and typically excites both longitudinal and flexural waves at the same time ${ }^{2,12}$. At the macroscopic scale, pass-band filters exploiting both longitudinal ${ }^{13}$ and torsional ${ }^{14}$ modes have been presented, and liquid sensing based on a cavity mode has been demonstrated ${ }^{15}$. Furthermore, longitudinal PnCs have been fabricated and measured in order to study the topological properties of bands and interface states ${ }^{16,17}$.

The properties of both finite and infinite longitudinal PnCs have been investigated in several theoretical papers. The transmission matrix method is the most common approach to deal with longitudinal PnCs made of piecewise linear segments ${ }^{18,19}$. For more complex geometries, expansion methods are employed ${ }^{20}$. Whatever the approach, the design of PnCs is typically focused on obtaining very large bandgap widths. However, attenuation in the frequency bandgap is also an important design parameter. For example, the efficiency of acoustic rejection in filtering applications depends on the attenuation that can be attained within the bandgap. In finite PnCs with small attenuation, an impractically long structure may be needed to reach the required attenuation. Having simple expressions for the attenuation can greatly simplify the evaluation of this trade-off in the synthesis of such structures. In this letter, we propose approximate expressions for the calculation of the attenuation constant of waves inside the bandgaps of longitudinal MEMS PnCs. To obtain adequate accuracy, two different models for small and large bandgaps are required. These asymptotic expressions are validated by comparison with results based on exact expressions for the dispersion relation. Results are discussed and compared with the ones of a similar study on flexural waves ${ }^{21}$.

\section{Model}

In a longitudinal PnC, the property of transmitting (or blocking) acoustic waves is contained in the dispersion function $k(\omega)$ giving the wavenumber $k$ as a function of the (angular) frequency. Propagating waves correspond to real wavenumbers, while imaginary wavenumbers give evanescent waves, i.e. blocked frequencies. The attenuation constant $\gamma(\omega)$ at a specific frequency $\omega$ inside a bandgap, i.e. the inverse of the attenuation length, can be then simply given as $\gamma=|\operatorname{Im}(k(\omega))|$. A simple method for estimating the attenuation capability of a bandgap is to consider the attenuation constant at the bandgap center, where its value is maximum or very close to it.

To derive compact expression for $\gamma$, we first define the geometry of the unit cell of a generic PnC, where the modulation of the acoustic contrast is obtained by regular perforations (Fig. 1, left). The PnC is composed by a generic number of repetitions of this unit cell, each of length $l$ along the $x$ axis. Each cell is composed by a full segment and a holed segment, and the related quantities are denoted by the $f$ and $h$ subscripts, respectively. Two dimensionless parameters define the cell geometry: $\alpha_{x}$ is the length of the full segment normalized to the cell length, and $\alpha_{y}$ is the area of the holed cross-section normalized to the full cross-section. The two parameters are comprised between 0 and 1 . The perforation along $y$ is best obtained with several small holes, so that we can assume that the holed segment behaves as an equivalent material of scaled density and stiffness, with a scale factor equal to $\alpha_{y}$. A further advantage of having several small holes is that the surface-to-volume ratio is increased, a property exploited in PnC-based sensors ${ }^{2,3}$. The cell geometry considered here is identical to the one presented in [21], where the propagation of flexural waves inside 1D PnCs was studied. For longitudinal waves, the structure is seen as a bar where deformations move the cross-sections along the main axis ${ }^{22}$, as show in the right side of Fig. 1. 


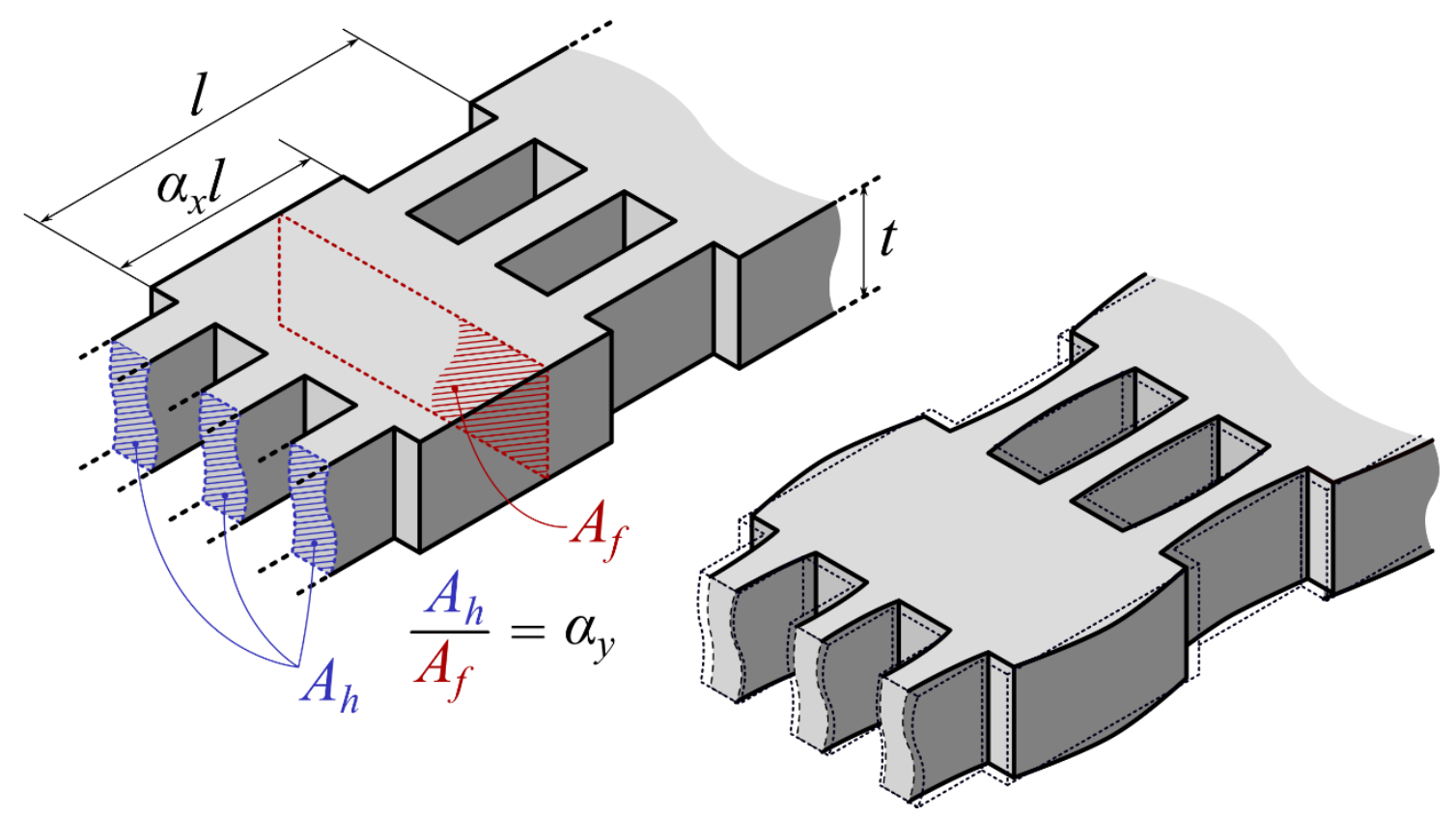

Figure 1. (left) Structure of the PnC cell and definition of $\alpha_{x}$ and $\alpha_{y}$; (right) sketch of its longitudinal deformation.

At any cross-section, the behavior of the structure is described by two parameters: its axial stiffness $(E A)$, i.e. the product of the Young's modulus $E$ and the area of the cross section $A$, and the translatory inertia $(\rho A)$, i.e. the product of the mass density $\rho$ and $A$. Because of the structure of the cell, both $(E A)$ and $(\rho A)$ are piecewise constant functions of $x$. The state of the PnC at the cross-section is described by a vector $\mathbf{s}(x)=[u(x) n(x)]^{\mathrm{T}}$, where $u(x)$ is the longitudinal displacement and $n(x)$ the axial force. We normalize all quantities by introducing the following definitions:

$$
\xi \equiv \frac{x}{l}, \quad U(\xi) \equiv \frac{1}{l} u(\xi l), \quad N(\xi) \equiv \frac{1}{(E A)_{f}} n(\xi l), \quad \mathbf{S}(\xi)=[U(\xi) \quad N(\xi)]^{T}
$$

where $\mathbf{S}(\xi)$ is a normalized state vector. With this normalization, the harmonic wave equation for longitudinal waves can be written as

$$
\frac{d}{d \xi}\left[\begin{array}{l}
U(\xi) \\
N(\xi)
\end{array}\right]=\left[\begin{array}{cc}
0 & \frac{(E A)_{f}}{(E A)} \\
-\Omega^{2} \frac{(\rho A)}{(\rho A)_{f}} & 0
\end{array}\right]\left[\begin{array}{l}
U(\xi) \\
N(\xi)
\end{array}\right]=\mathbf{D} \cdot \mathbf{S}(\xi)
$$

where $\mathbf{D}$ is the dynamic matrix of the system. The normalized frequency $\Omega^{18}$ has also been introduced:

$$
\Omega \equiv \frac{\omega}{\omega_{0}}, \quad \omega_{0} \equiv \frac{c}{l}, \quad c \equiv \sqrt{\frac{E}{\rho}}
$$

with $\omega$ being the frequency. The quantity $c$ is the speed of longitudinal waves inside the bar (which, in our case, is independent of $x$ ). The matrix $\mathbf{D}$ assumes two different values in the two segments of the cell:

$$
\boldsymbol{D}_{\boldsymbol{f}}=\left[\begin{array}{cc}
0 & 1 \\
-\Omega^{2} & 0
\end{array}\right], \quad \boldsymbol{D}_{\boldsymbol{h}}=\left[\begin{array}{cc}
0 & 1 / \alpha_{y} \\
-\Omega^{2} \alpha_{y} & 0
\end{array}\right] .
$$

Equation (2) can be solved for the transmission matrix $\mathbf{T}$ of the unit cell, defined by the relation $\mathbf{S}(\xi+1)=$ $\mathbf{T} \cdot \mathbf{S}(\xi)$. Its value is:

$$
\mathbf{T}=\exp \left[\alpha_{x} \boldsymbol{D}_{\boldsymbol{f}}(\Omega)\right] \exp \left[\left(1-\alpha_{x}\right) \mathbf{D}_{\boldsymbol{h}}(\Omega)\right]
$$


Where $\exp (\mathbf{M})$ is the matrix exponential of $\mathbf{M}$. The two eigenvalues of $\mathbf{T}$ are the inverse of each other, and can be written as $e^{ \pm i K(\Omega)}$, with $K(\Omega)$ being the dispersion function. Straightforward passages (see Appendix A for details) give:

$$
K=-i \ln \left(\frac{1}{2}\left(-I_{L}+\sqrt{I_{L}^{2}-4}\right)\right)
$$

where $I_{L}$ is an invariant of the matrix (equal to minus the trace of $\mathbf{T}$ ). Its expression is:

$$
I_{L}\left(\alpha_{x}, \alpha_{y}, \Omega\right)=-\operatorname{tr}(\mathbf{T})=-2 \cos \left(\alpha_{x} \Omega\right) \cos \left(\left(1-\alpha_{x}\right) \Omega\right)+\frac{1+\alpha_{y}^{2}}{\alpha_{y}} \sin \left(\alpha_{x} \Omega\right) \sin \left(\left(1-\alpha_{x}\right) \Omega\right) .
$$

The expression of $I_{L}$ is also invariant under the substitution $\alpha_{x} \rightarrow 1-\alpha_{x}$. As a result, we limit our study to $\alpha_{x} \in[0,1 / 2]$. A frequency is inside a bandgap if the corresponding wavenumber has nonzero imaginary part. It is immediate to verify from (6) that this happens in the (countably infinite) intervals of $\Omega$ where $\left|I_{L}\right|>2$. The sign in the definition of $I_{L}$ was chosen (consistently with what we did for flexural crystals ${ }^{21}$ ) so that frequencies within an odd bandgap have $I_{L}>2$, those in even bandgaps have $I_{L}<-2$. To compute the attenuation at the center of any of these intervals, it is convenient to define a normalized attenuation constant $\Gamma_{n}$ as:

$$
\Gamma_{n}=\frac{\left|\operatorname{Im}\left(K\left(\Omega_{n}\right)\right)\right|}{\Omega_{n}} .
$$

The attenuation constant $\gamma$ is then

$$
\gamma=\left|\operatorname{Im}\left(k\left(\omega_{n}\right)\right)\right|=\left|\frac{\operatorname{Im}\left(K\left(\Omega_{n}\right)\right)}{l}\right|=\frac{\left|\operatorname{Im}\left(K\left(\Omega_{n}\right)\right)\right|}{\Omega_{n}} \omega_{n} \sqrt{\frac{\rho}{E}}=\Gamma_{n} \frac{\omega_{n}}{c}
$$

where $\omega_{n}$ is the center frequency of the $n^{\text {th }}$ bandgap, and $\Omega_{n}$ is its normalized value. In the following, analytical approximated expressions of the normalized attenuation constant $\Gamma_{n}$ are derived and discussed.

For comparison with these asymptotic expressions, accurate numerical values of the attenuation were calculated with the same method proposed in ${ }^{21}$. Briefly, for any point $\left(\alpha_{x}, \alpha_{y}\right)$ of the design space, the bandgap edges can be extracted by numerically solving for the roots of $I_{L}\left(\alpha_{x}, \alpha_{y}, \Omega\right)= \pm 2$. Once that the bandgap edges (and thus their central frequencies) are known, the normalized attenuation constant for each bandgap can be calculated by using (6)-(8). The numerical values of $\Gamma_{n}$ for the first three bandgaps are shown as red dotted curves in the contour plots of Fig. 2. In the rest of the paper, we use these numerical values to assess the accuracy of our approximated models.
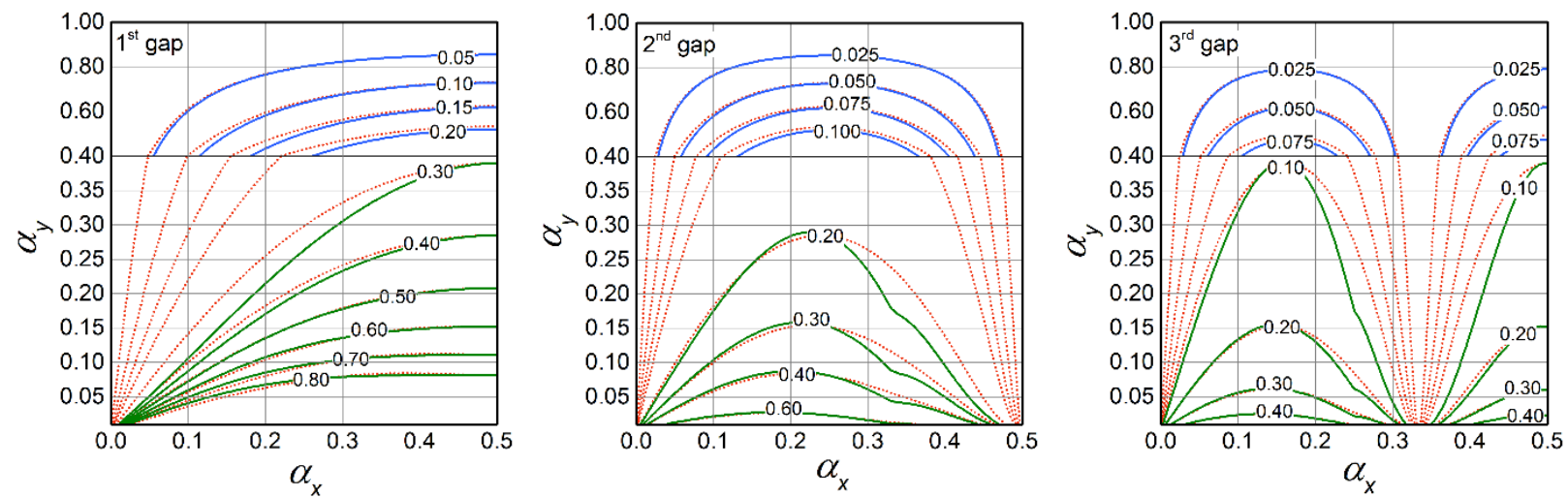

Figure 2. Values of the normalized attenuation $\Gamma_{n}$ for the first, second and third bandgap (left to right). Red dotted lines are numerical values, blue lines the approximation for small bandgaps, green lines the approximation for large bandgaps. 


\section{Discussion}

\subsection{Large bandgap approximation}

While a small contrast between the two segments (i.e. an $\alpha_{y}$ close to one) always leads to narrow bandgaps, the opposite (i.e., large bandgaps for large contrasts) is not necessarily true. This can be verified with the aid of Fig. 3, which shows the borders of the first three bandgaps (computed with the method described above) as a function of $\alpha_{x}$ for three different values of $\alpha_{y}$, namely $0.3,0.1$, and 0.033 . The graph clearly shows that there are critical values of $\alpha_{x}$ for which the bandgaps disappear, irrespective of the value of $\alpha_{y}$. The value $\alpha_{x}=0$ (and, symmetrically, $\alpha_{x}=1$ ) correspond to the trivial case of homogeneous rods without discontinuities, where acoustic propagation is unrestricted. The other critical values of $\alpha_{x}$ are located at the points of geometrical phase inversion ${ }^{16}$.

To explore the case of large bandgaps, we then assume the necessary, though not sufficient, condition, that $\alpha_{y} \ll 1$. We note that in the limit $\alpha_{y} \rightarrow 0$, the equation for the bandgap borders, $\left|I_{L}\right|=2$ tends to:

$$
\sin \left(\alpha_{x} \Omega\right) \sin \left(\left(1-\alpha_{x}\right) \Omega\right)=0,
$$

whose solutions are, trivially, the two families of hyperbolas

$$
\Omega=\frac{q}{1-\alpha_{x}} \pi, \quad \Omega=\frac{p}{\alpha_{x}} \pi \quad q, p=1,2,3, \ldots
$$

These hyperbolas are plotted in Fig. 3 as solid black lines. The inversion points stand at the intersection of these hyperbolas which, for the $n^{\text {th }}$ bandgap, take place for $\alpha_{x}=m / n$, with $m=1,2, \ldots(n-1)$. For $\alpha_{y} \rightarrow 0$, the bandgaps expand to fill all frequencies and the propagation bands reduce to single points. A theoretical upper limit for the bandgap width can be obtained as the difference of two adjacent hyperbolas. This analysis shows that a best-case approximation for the normalized fractional bandwidth is $2 / n$, with $n$ as the bandgap order, i.e. large fractional bandwidths are progressively harder to obtain at higher orders.

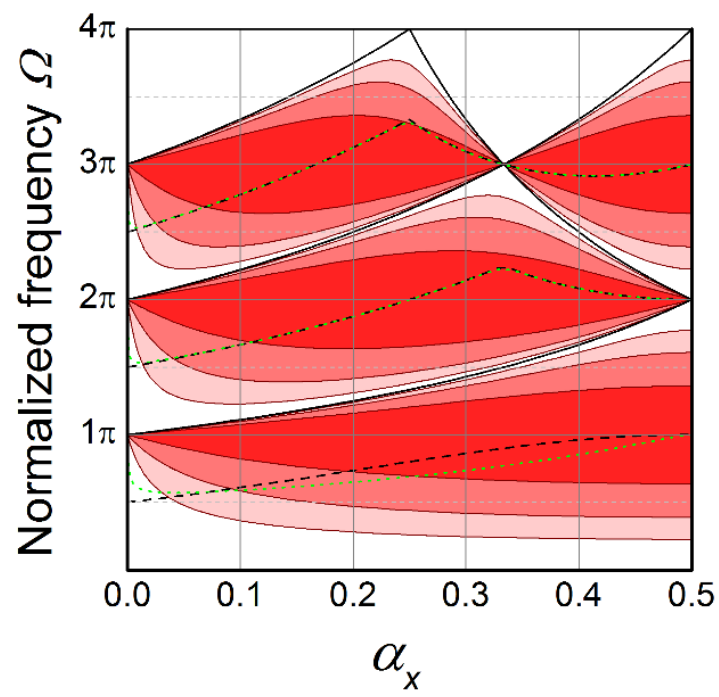

Figure 3. First three bandgaps at three different $\alpha_{y}$ values (0.033, 0.1, 0.3 for progressively darker red shades) as a function of $\alpha_{x}$. Solid black lines mark the asymptotic bandgap borders (11), dashed black lines the approximate bandgap centers ((14)-(15)), green dotted lines the numerical bandgap centers for $\alpha_{y}=0.001$. 
Under the assumption of large bandgaps, the modulus of the invariant at the bandgap center $I_{L}\left(\Omega_{\mathrm{n}}\right)$ is also large (i.e. much larger than 2). Under this assumption, the wavenumber (6) can be approximated to

$$
K= \begin{cases}i \ln \left(\left|I_{L}\right|\right) & \text { even bandgaps, } \\ \pi-i \ln \left(\left|I_{L}\right|\right) & \text { odd bandgaps. }\end{cases}
$$

The attenuation $\Gamma_{n}$ can then be written, from (7) and (12), and under the further assumption $\alpha_{y} \ll 1$, as

$$
\Gamma_{n}=\frac{1}{\Omega_{n}} \ln \left(\frac{\left|\sin \left(\alpha_{x} \Omega_{n}\right) \sin \left(\left(1-\alpha_{x}\right) \Omega_{n}\right)\right|}{\alpha_{y}}\right) .
$$

To evaluate (13), estimations of the $\Omega_{n}$ 's are required. The simplest choice is to approximate $\Omega_{n}$ with its value for $\alpha_{y} \rightarrow 0$, which can be easily computed as the average of the asymptotic bandgap borders as determined by (11), leading to the following expressions:

$$
\Omega_{2}=\left\{\begin{array}{ll}
\frac{3 \pi}{2\left(1-\alpha_{x}\right)} & 0 \leq \alpha_{x}<1 / 3 \\
\frac{\pi}{2 \alpha_{x}\left(1-\alpha_{x}\right)} & \frac{1}{3} \leq \alpha_{x} \leq 1 / 2
\end{array} \quad \Omega_{3}=\left\{\begin{array}{cc}
\frac{5 \pi}{2\left(1-\alpha_{x}\right)} & 0 \leq \alpha_{x}<1 / 4 \\
\frac{\pi\left(1+\alpha_{x}\right)}{2 \alpha_{x}\left(1-\alpha_{x}\right)} & 1 / 4 \leq \alpha_{x} \leq 1 / 2
\end{array}\right.\right.
$$

This is a very good approximation for bandgap borders higher than one, as shown in Fig. 3 by the comparison between the approximated solution (solid green lines) and numerically computed values (dotted red lines). However, for the first bandgap, the fact that the lower border reaches zero for $\alpha_{y} \rightarrow 0$ leads to an excessive error with respect to the numerical case. In this case we choose a different expression:

$$
\Omega_{1} \approx \frac{\pi}{1+\left(1-2 \alpha_{x}\right)^{2}}
$$

which captures more accurately the actual behavior for $\alpha_{x}$ close to $1 / 2$. Equation (15) is also plotted as a dashed black line in Fig. 3. Substituting the approximate expressions for $\Omega_{n}$ into (13), we finally compute the attenuation as a function of both $\alpha_{x}$ and $\alpha_{y}$. The corresponding curves are plotted in Fig. 2 as solid green lines. Again, a close approximation of actual numerical values is obtained for large bandgaps, i.e. for small $\alpha_{y}$ and far from the values of $\alpha_{x}$ where the bandgaps close, i.e. in the homogeneous limit $\left(\alpha_{x}=0, \alpha_{x}=1\right)$, and at inversion points $\left(\alpha_{x}=m / n\right.$, with $m, n$ integers, $\left.m<n\right)$.

\subsection{Small bandgap approximation}

On the other hand, a small acoustic contrast always leads to small bandgaps. In this case, we determine that the normalized attenuation constant $\Gamma_{n}$ is, in the limit of infinitesimally small deviations from the homogenous rod, proportional to the fractional bandwidth:

$$
\Gamma_{n} \approx \frac{1}{2} \frac{\Delta \Omega_{n}}{\Omega_{n}}
$$

where $\Delta \Omega_{n}$ is the normalized width of the bandgap (a proof of (16) is given in Appendix B). Using this approximation, the problem of estimating the attenuation constant is transformed to the one of estimating bandgap edges. This problem can be solved for small deviations from the homogenous case $\left(\alpha_{y}=1\right)$ by using the perturbation method adopted by Nielsen and Sorokin ${ }^{19}$. By setting $\alpha_{y}=1-\epsilon$ and expanding up to the $3^{\text {rd }}$ order with respect to $\epsilon$, the following relationship is obtained:

$$
\frac{\Delta \Omega_{n}}{\Omega_{n}} \approx 2 \epsilon\left|\sin \left(n \pi \alpha_{x}\right)\right| \frac{1+\frac{\epsilon}{2}+\frac{1}{12} \epsilon^{2}\left(4+2 \cos \left(2 n \pi \alpha_{x}\right)-3\left(1-\alpha_{x}\right) \alpha_{x}\left(1+3 \cos \left(2 n \pi \alpha_{x}\right)\right)\right)}{n \pi-\frac{\epsilon^{2}}{4}\left(1-2 \alpha_{x}\right) \sin \left(2 n \pi \alpha_{x}\right)} .
$$


The corresponding analytical values for the first three gaps are plotted (blue solid curves) in Fig. 2 against $\alpha_{x}$ and $\alpha_{y}$. A comparison with the numerically computed values (red dotted curves) show that there is good agreement up to relatively high values of contrast, with $0.4<\alpha_{y}<1$ being a reasonable range. A relationship similar to (16), i.e. $\Gamma_{n} \approx \Delta \Omega_{n} / 4 \Omega_{n}$ holds for flexural $\mathrm{PnCs}^{21}$. An important consequence of this fact is that, for the same fractional bandwidth $\Delta \Omega_{n} / \Omega_{n}$, the normalized in-gap attenuation in longitudinal PnCs is about twice as large as the one in flexural PnCs. For $\alpha_{y}<0.4$, this approximation progressively fails, and the attenuation is larger than implied by (16). This is in contrast with the flexural case, where the equivalent approximation holds down to very low values of $\alpha_{y}$.

\subsection{Comparison with flexural crystals}

In the limit of small bandgaps, an insightful comparison of the attenuations can be established between flexural and longitudinal crystals. Specifically, it is interesting to compare the attenuation for PnCs with the same fractional bandwidth, to assess how a flexural and longitudinal $\mathrm{PnC}$ with the same bandgap compare from the point of view of attenuation. For flexural waves, a small bandgap approximation for the attenuation (corresponding to (16) for longitudinal waves) exists ${ }^{21}$ :

$$
\Gamma_{n F} \approx \frac{1}{4} \frac{\Delta \Omega_{n}}{\Omega_{n}}
$$

where $\Gamma_{n F} \doteq\left|\operatorname{Im}\left(K_{f}\left(\Omega_{n F}\right)\right)\right| / \sqrt{\Omega_{n F}}$ is the normalized attenuation constant for flexural waves ${ }^{21}$. By equating the fractional bandwidths in (16) and (18), and exploiting the normalization coefficients, a denormalized expression is found:

$$
\gamma_{F}=\frac{3^{1 / 4}}{\sqrt{2}} \sqrt{\frac{c}{t \omega_{n}}} \gamma_{L}
$$

where $t$ is the thickness and $\gamma_{F}$ and $\gamma_{L}$ are the attenuations per unit length for flexural and longitudinal waves, respectively. A insightful way to present this result is to define $t_{e}$ as the thickness at which the two are equal $\left(\gamma_{F}=\gamma_{L}\right)$, compute it from (19) and plot it as a function of the target central frequency $\omega_{n}$. The expression for $t_{e}$, which also depend on the speed of waves $c$ in the material, is: 


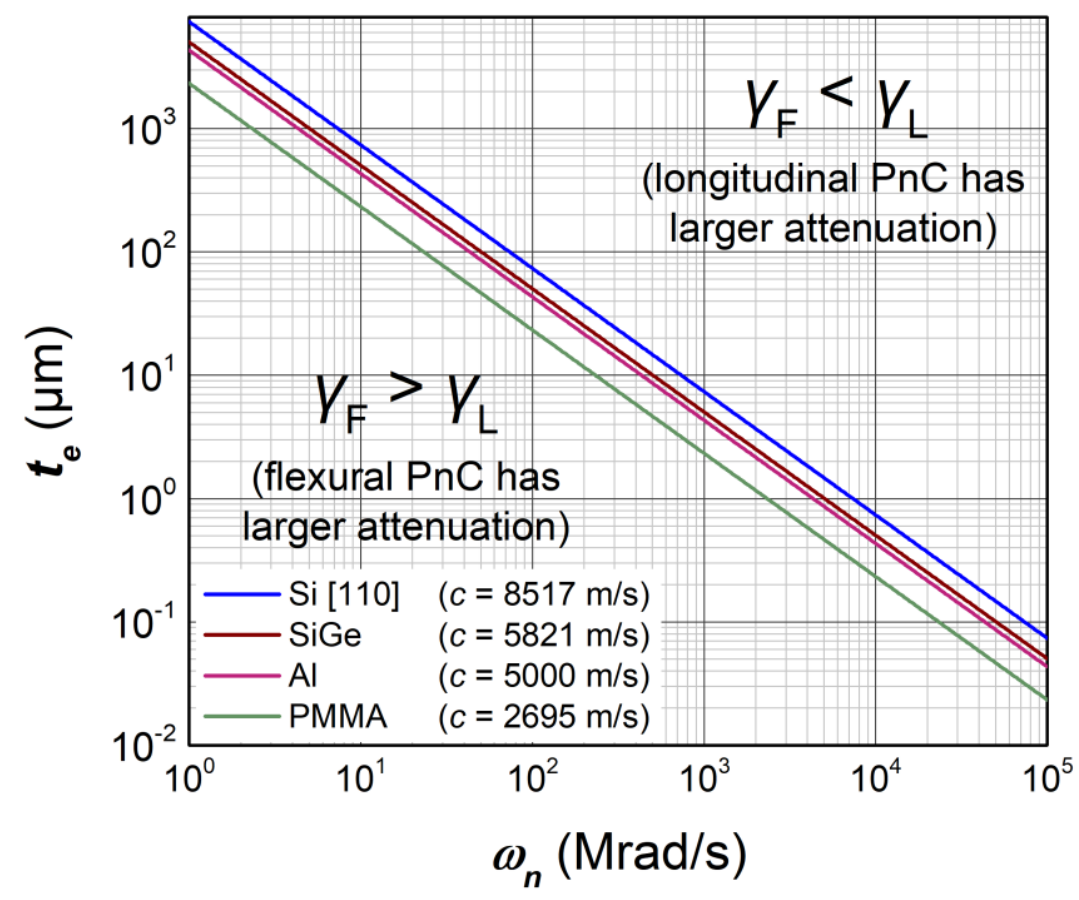

Figure 4. Structural thickness giving equal attenuation for flexural and longitudinal waves $\left(\gamma_{F}=\gamma_{L}\right)$ as a function of the target central frequency of the bandgap for different structural materials.

$$
t_{e}=\frac{\sqrt{3}}{2} \frac{c}{\omega_{n}}
$$

A family of lines describing various materials used in applications (monocrystalline silicon, polycrystalline silicon-germanium, aluminum, and poly methyl methacrylate or PMMA) are shown in Fig. 4. Below the lines there are the design points where flexural attenuation is larger than longitudinal attenuation, the opposite is true above the lines. Moving towards the $\mathrm{GHz}$ domain (which is of interest in radio-frequency filtering applications), data show that a longitudinal design offers a better rejection.

\section{Conclusions}

In this work, we developed simplified analytical expressions for the in-gap attenuation constant of longitudinal N/MEMS PnCs for the two cases of large bandgap and small bandgap structures and validated these expressions against the attenuation constant obtained numerically from the transmission matrix theory. We then showed that, for small bandgaps, the attainable attenuation essentially only depends on the bandgap widthto-center ratio (i.e., the fractional bandwidth). For large bandgaps, larger attenuations can be obtained at relatively small acoustic constraints (which are technologically easier to obtain), as long as the design avoids structures close the inversion points for the propagation of longitudinal waves. Furthermore, we compared the attenuation of longitudinal and flexural waves at fixed fractional bandwidth, and showed that two different domains exist for better attenuation between the two propagation modes, with longitudinal PnCs favored at high frequency and large device thickness. All these results will prove useful in efficient synthesis of microto nanoscale PnCs.

\section{Appendix A}


Wave number expression. The characteristic polynomial $P(\lambda)$ of the transmission matrix $\mathbf{T}$ of the unit cell (see equation (5)) can be written as

$$
P(\lambda)=\lambda^{2}+I_{L} \lambda+1
$$

where $I_{L}$ is the invariant of equation (7), equal to minus the trace of $\mathbf{T}$. The two eigenvalues are obtained by solving $P(\lambda)=0$, giving

$$
\lambda_{1,2}=\frac{1}{2}\left(-I_{L} \pm \sqrt{I_{L}^{2}-4}\right) .
$$

By rewriting such eigenvalues in exponential form $\lambda_{1,2}=e^{ \pm i K}$, where $K$ is Bloch wavenumber describing the propagation of longitudinal waves inside the $\mathrm{PnC}$, we obtain (6).

\section{Appendix B}

Relationship between fractional bandwidth and normalized attenuation. Following a similar derivation of the one presented in [21], we demonstrate equation (16) in the limit of small deviations from an homogeneous rod, i.e. a bar with uniform cross section and material properties along its axis. Such a structure has a linear dispersion relation of the form

$$
K= \pm \Omega
$$

Without loss of generality, we assume that a periodicity is introduced in a homogeneous rod by a perturbation parameter $\delta \alpha$, with $\delta \alpha=0$ being the homogeneous case. A change in $\alpha$ can be related to a change in geometry, density, stiffness or their combination. The condition for $K$ to be real, i.e., for $\Omega$ to be outside of a bandgap is

$$
-2 \leq I_{L}(\delta \alpha, \Omega) \leq 2 .
$$

We limit our derivation to the case of odd bandgaps were $I_{L}(\delta \alpha, \Omega)>2$. In the homogeneous case (S.4) is always verified, but $I_{L}$ assumes the value 2 in a numerable set of values $\Omega_{n 0}$ of the frequency. A perturbation of the homogeneity (i.e., a change in $\delta \alpha$ ) opens a bandgap around any of these frequencies. We define

$$
\delta I_{L}(\delta \alpha, \delta \Omega)=I_{L}\left(\delta \alpha, \Omega_{n 0}+\delta \Omega\right)-2 .
$$

Inside the opening bandgap, $\delta I_{L}$ is always positive. For small $\delta I_{L}$ and odd bandgaps, (6) can be approximated with its first order Taylor series as

$$
K \approx n \pi \pm i \sqrt{\delta I_{L}(\delta \alpha, \delta \Omega)}, n \text { odd. }
$$

It is immediate that in the homogeneous case no bandgap opens, and thus $\delta I_{L}(0, \delta \Omega) \leq 0$. Furthermore, as a change in $\delta \alpha$ opens the bandgap, $\delta I_{L}(\delta \alpha, 0) \geq 0$. The point $(0,0)$ is then a saddle point for $\delta I_{L}(\delta \alpha, \delta \Omega)$. We can now write the second order series expansion of $\delta I_{L}$ around the point $(0,0)$

$$
\delta I_{L}(\delta \alpha, \delta \Omega) \approx \frac{1}{2} I_{L, \alpha \alpha} \delta \alpha^{2}+I_{L, \alpha \Omega} \delta \alpha \delta \Omega+\frac{1}{2} I_{L, \Omega \Omega} \delta \Omega^{2}
$$

where we used comma notation for partial derivatives, and exploited the fact that, because of the existence of the saddle point, first order derivatives are zero. Substituting (B.5) in (B.4)

$$
K \approx n \pi \pm i \sqrt{\frac{1}{2} I_{L, \alpha \alpha} \delta \alpha^{2}+I_{L, \alpha \Omega} \delta \alpha \delta \Omega+\frac{1}{2} I_{L, \Omega \Omega} \delta \Omega^{2}}, n \text { odd }
$$

Equation (B.6) is then an approximate expression for the dispersion relation near the opening bandgap, from which we can determine the normalized bandgap width $\Delta \Omega_{n}$, its center $\Omega_{n}$, and normalized attenuation $\Gamma_{n}$. Straightforward calculations give: 


$$
\begin{gathered}
\Delta \Omega_{n}{ }^{2}=4 \frac{I_{L, \alpha \Omega}{ }^{2}-I_{L, \alpha \alpha} I_{L, \Omega \Omega}}{I_{L, \Omega \Omega}{ }^{2}} \delta \alpha^{2} \\
\Omega_{n}=\Omega_{n 0}-\frac{I_{L, \alpha \Omega}}{I_{L, \Omega \Omega}} \delta \alpha \approx \Omega_{n 0} \\
\Gamma_{n}{ }^{2}=\frac{\left|\operatorname{Im}\left(K_{2}\left(\Omega_{n}\right)\right)\right|^{2}}{\Omega_{n}^{2}}=\frac{I_{L, \alpha \Omega}{ }^{2}-I_{L, \alpha \alpha} I_{L, \Omega \Omega}}{2\left|I_{L, \Omega \Omega}\right| \Omega_{n 0}^{2}} \delta \alpha^{2} .
\end{gathered}
$$

The squared ratio between $\Gamma_{n}$ and the fractional bandwidth can thus be written as:

$$
\left(\frac{\Gamma_{n}}{\frac{\Delta \Omega_{n}}{\Omega_{n}}}\right)^{2}=\frac{1}{8}\left|I_{L, \Omega \Omega}\right| .
$$

The values of $\Omega_{n 0}$ and $I_{L, \Omega \Omega}$ can be determined by equating (B.1) and (B.6) in the homogeneous $(\delta \alpha=0)$ case:

$$
K(0, \delta \Omega)= \pm \Omega_{n 0} \pm \delta \Omega=n \pi \pm i \sqrt{\left(I_{L, \Omega \Omega} / 2\right) \delta \Omega^{2}} .
$$

The left and right sides of (B.11) are now two approximations of the same dispersion relation. Comparison of the two gives:

$$
\Omega_{n 0}=n \pi, \quad I_{L, \Omega \Omega}=-2 .
$$

Finally, substitution of (B.12) in (B.10) gives

$$
\Gamma_{n}=\frac{1}{2} \frac{\Delta \Omega_{n}}{\Omega_{n}} .
$$

\section{REFERENCES}

${ }^{1}$ M.I. Hussein, M.J. Leamy, and M. Ruzzene, Appl. Mech. Rev. 66, 40802 (2014).

${ }^{2}$ L. Luschi and F. Pieri, in Proc. 2015 18th AISEM Annu. Conf. AISEM 2015 (2015).

${ }^{3}$ B. Figeys, R. Jansen, S. Severi, B. Nauwelaers, H.A.C. Tilmans, and X. Rottenberg, in 2015 Transducers 2015 18th Int. Conf. Solid-State Sensors, Actuators Microsystems (IEEE, 2015), pp. 141-144.

${ }^{4}$ F.-C. Hsu, J.-C. Hsu, T.-C. Huang, C.-H. Wang, and P. Chang, Appl. Phys. Lett. 98, 143505 (2011).

${ }^{5}$ D. Hatanaka, I. Mahboob, K. Onomitsu, and H. Yamaguchi, Nat. Nanotechnol. 9, 520 (2014).

${ }^{6}$ M. Eichenfield, J. Chan, R.M. Camacho, K.J. Vahala, and O. Painter, Nature 462, 78 (2009).

${ }^{7}$ M. Maldovan, Nat. Mater. 14, 667 (2015).

${ }^{8}$ P.E. Hopkins, C.M. Reinke, M.F. Su, R.H. Olsson, E.A. Shaner, Z.C. Leseman, J.R. Serrano, L.M.

Phinney, and I. El-Kady, Nano Lett. 11, 107 (2011).

${ }^{9}$ S. Mohammadi and A. Adibi, J. Microelectromechanical Syst. 21, 379 (2012).

${ }^{10}$ L. Luschi and F. Pieri, in Procedia Eng. (2012), pp. 1101-1104.

${ }^{11}$ T. Mattila, J. Kiihamäki, T. Lamminmäki, O. Jaakkola, P. Rantakari, A. Oja, H. Seppä, H. Kattelus, and I. Tittonen, Sensors Actuators A Phys. 101, 1 (2002).

${ }^{12}$ C. Maurini, J. Pouget, and F. Dell'Isola, Comput. Struct. 84, 1438 (2006).

${ }^{13}$ H.J. Lee, J.K. Lee, and Y.Y. Kim, J. Sound Vib. 353, 58 (2015).

${ }^{14}$ I.K. Lee, H.M. Seung, and Y.Y. Kim, J. Sound Vib. 355, 86 (2015). 
${ }^{15}$ R. Lucklum, M. Ke, and M. Zubtsov, Sensors Actuators B Chem. 171-172, 271 (2012).

${ }^{16}$ M. Xiao, G. Ma, Z. Yang, P. Sheng, Z.Q. Zhang, and C.T. Chan, Nat. Phys. 11, 240 (2015).

${ }^{17}$ Y.-X. Fan, T.-Q. Sang, T. Liu, L.-L. Xu, and Z.-Y. Tao, Sci. Rep. 7, 44381 (2017).

${ }^{18}$ M.I. Hussein, G.M. Hulbert, and R.A. Scott, J. Sound Vib. 289, 779 (2006).

${ }^{19}$ R.B. Nielsen and S.V. Sorokin, J. Sound Vib. 353, 135 (2015).

${ }^{20}$ A.J. Hull and B.A. Cray, J. Acoust. Soc. Am. 136, 2196 (2014).

${ }^{21}$ L. Luschi, F. Pieri, and G. Iannaccone, IEEE Trans. Electron Devices 63, (2016).

${ }^{22}$ P. Hagedorn and A. DasGupta, Vibrations and Waves in Continuous Mechanical Systems (2007). 